\title{
Position and role of the chief knowledge officer in South Africa - a discrepancy between theory and practice?
}

\author{
KC Chauke \\ National Research Foundation \\ kholane@nrf.ac.za \\ M.M.M. Snyman \\ Department of Information Science \\ University of Pretoria \\ msnyman@postino.up.ac.za
}

\section{Contents}

1. Background

2. Methodology

3. Concept of chief knowledge officer (CKO)

4. Role and responsibilities of the CKO

5. Knowledge and skills of the CKO

6. Findings of the interviews

7. Analysis of the findings

8. Conclusion

9. References

\section{Background}

The knowledge economy has become a reality for many enterprises. In the knowledge economy, it is not products and services that give enterprises a competitive advantage, but how well they manage their knowledge, in other words, what they know and whether they know how to do new things quickly. Bontis (2002:1) states that knowledge management how an organization makes use of its intellectual capital - is one of the greatest challenges facing enterprises today. However, knowledge management requires an individual with specific knowledge and skills to champion the concept of knowledge management and spearhead the enormous challenges needed to overcome inherent obstacles to the free flow of knowledge within an enterprise. A new role with the title of chief knowledge officer (CKO) has emerged to manage this unique organizational asset - intellectual capital.

It is difficult to estimate how many positions exist for CKOs, as most of these positions are internal appointments and few advertisements for this position appear in the press. (Bontis 2002:3; TFPL 1999a:4; Tiwana 2000:405) Earl and Scott (2001) estimate that there are 
approximately 50 CKOs in the world. A few positions for CKOs have also emerged in South Africa. However, much confusion still exists about who and what the CKO is, what his or her responsibilities are and what skills he or she should possess. From an analysis of job advertisements for knowledge managers in South Africa, it became clear that, although an awareness of the need for a person to manage intellectual capital at a strategic level does exist in South African enterprises, a comprehensive understanding of the position and role of the CKO is lacking (Snyman 2001).

The aim of this research was to investigate the South African business world's perspective of the role and responsibilities of the CKO and to analyse these findings against a theoretical background. To achieve this aim, the following aspects were investigated:

- The concept of CKO

- The role and responsibilities of the CKO

- Qualifications, knowledge and skills required for the position of CKO

- How persons in CKO positions in South Africa weigh up against requirements in the literature.

\section{Methodology}

Although CKO positions have been in existence for sometime internationally or abroad, they are not very common in South Africa; hence very few enterprises in South Africa have people designated to work in this capacity. Taking the definition of a CKO (see below) into consideration and working through the mailing list of the Knowledge Management Society of South Africa as well as analysing advertisements for knowledge managers, which appeared in three weekly newspapers with the highest circulation figures in South Africa during 2000 and 2001, only three CKOs were identified. The business world's perspective of the CKO was determined during semi-structured interviews with the three persons in the position of CKO. During the interviews, which lasted between two to three hours, it was attempted to collect information on their position and role as CKO in their enterprise and the knowledge and skills required for the position. The findings of the interviews were studied and analysed against the theoretical background obtained during a literature study.

\section{Concept of chief knowledge officer (CKO)}

The CKO is a recent phenomenon created by enterprises to lead and promote the knowledge management agenda and to help manage a unique organizational asset, namely intellectual capital (IC). The CKO concept is rooted in the realization that enterprises can no longer expect that the products and services that made them successful in the industrial age will keep them viable in the future (Herschel and Nemati 2000). The challenges and pressures of a rapidly changing global and knowledge-based economy make tacit and explicit knowledge vital to organizations. Therefore, the CKO position has emerged as a top management responsibility, comprising of leadership and change management. These pioneering individuals have been given the unenviable task of channelling an enterprise's knowledge into initiatives that are expected to become a source of competitive advantage (Bontis 2002:1). They generally provide the focus and drive required to steer knowledge management programmes through critical stages and facilitate planning and development (TFPL 1999a and 1999b).

Earl and Scott (2000) define the CKO as a senior executive leading a knowledge 
management initiative and who has 'knowledge' in his or her job title. The chief executive officer (CEO) appoints these executives and they report to the CEO. The aim of the CKO is to initiate knowledge management and to ensure that the philosophy and practice became embedded in the enterprise (Earl and Scott 2000:196). However, they do not see it as a longterm position. Skyrme (1997:1) describes the CKO as 'a senior executive who is responsible for ensuring that an organization maximises the value it achieves through one of its most important assets - knowledge'. The key role that these individuals play is to make the knowledge management system and processes an integral part of the operations of the enterprise (Tiwana 2002:395).

CKOs come in many guises and with many titles such as director of knowledge management, strategic knowledge manager, director of intellectual capital, chief knowledge officer an executive director: knowledge management and strategy. For the purposes of this article the title chief knowledge officer (CKO) is used. A CKO is described as a senior executive with the word 'knowledge' in his/her title and who is responsible for developing and implementing knowledge management initiatives in the enterprise.

The challenge for the CKO is to develop strategies for converting tacit knowledge into explicit knowledge, which in a way means, as Nonaka (1998:31) states 'finding a way to express the inexpressible'. The CKO has to promote 'the development and application of tacit, explicit, and embedded intellectual capital; that is, leveraging personal understanding, organizational action capabilities, and other intellectual assets to attain the enterprise's ultimate goals, such as ascertaining profitability, ensuring long-term viability, or delivering quality services' (Wiig 2000:13-14).

It is therefore clear that the CKO position is a very important position in enterprises today. It involves designing and implementing a knowledge management strategy and initiatives to support the strategic direction of the enterprise.

\section{Role and responsibilities of the CKO}

Most enterprises are still defining their knowledge management roles (TFPL 1999:3). According to Gamble and Blackwell (2001:97), the role and the responsibilities of the CKO are still evolving. Earl (1999:4) states that the role of the CKO is so immature that there is no job specification. Therefore, CKOs have to work out an agenda for themselves. One of the 20 CKOs included in Earl and Scott's study rightfully mentioned that 'most CKOs are on a vertical learning curve about managing knowledge' (Tiwana 2000:396).

Knowledge management in every enterprise is unique and the knowledge management roles reflect this (TFPL 1999a:4). Therefore, the role and responsibilities of a CKO in an enterprise is interpreted differently depending on the needs and environment of the enterprise. However, during the literature study a number of generic roles and corresponding responsibilities of the CKO were identified. These roles and responsibilities are reflected in Figure 1.

Figure 1 Role and responsibilities of the CKO 


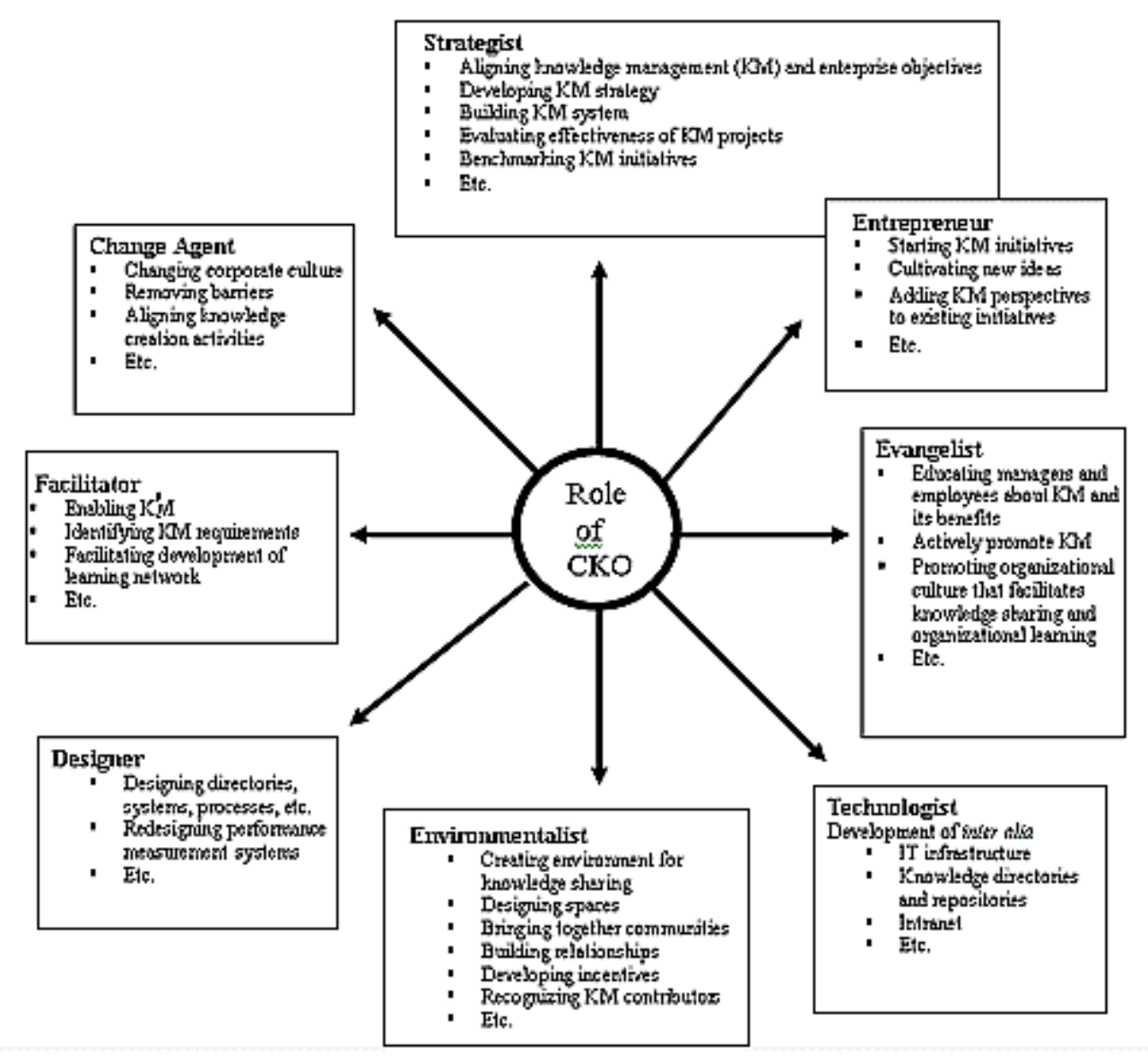

The CKO must be a strategist that has a clear vision of where the enterprise is going and how knowledge management can support the mission and objectives of the enterprise. It becomes the responsibility of the CKO to develop a strategy that dictates how an enterprise handles its intellectual assets and to build a knowledge management system to support the business processes. The most challenging issue for the CKO is that most of the knowledge is tacit in nature, and is embodied in people. The challenge for the CKO is therefore to strategize ways and actions to capture the tacit knowledge and to make it available for knowledge re-use and innovation (Allee 1997). The CKO also has to evaluate the effectiveness of knowledge management projects and their contribution to the mission and objectives of the enterprise. Furthermore, they have to benchmark the knowledge management efforts with those of other enterprises (Neilson 2002).

Because the CKO is such a new position, there are no historical models for a CKO. It is often expected of the CKO to act as an entrepreneur and to start a new knowledge management activity, capability or function. To fulfil this role he or she has to bring in new ideas, cultivate them, and listen to other people's ideas and back them if they make sense and fit the knowledge vision (Earl and Scott 2000:198). The CKO should also hold on to existing initiatives where these can add a knowledge management perspective.

Management support is critical for the long-term success of any knowledge management strategy, and showing managers the value of knowledge management is a prerequisite for the successful management of knowledge. On the other hand, users must not only know about the use and value of knowledge management, they also must be shown what's in it for them (Tiwana 2001:396). As an evangelist, the major challenge of the CKO is to convince the two distinct groups (management and the knowledge workers who will actually use knowledge management as part of their work) of the value of knowledge management. Therefore, it is expected of the CKO to create an awareness of knowledge management and to actively promote knowledge management, its adoption and use. This involves creating a common 
understanding of what knowledge management is, encouraging and supporting people, and lobbying for resources (Morris, Meed and Svensen 1996:112). As an evangelist, the CKO also has to promote an organizational culture that facilitates knowledge sharing and organizational learning (Neilson 2002). This can be done by making presentations, issuing publications and arranging conferences and seminars on knowledge management.

Technology channels are the socio-technical networks that help move knowledge around the organization in an efficient manner. Channels must be developed for the distribution of explicit knowledge and for the sharing of tacit knowledge. Technology channels and their choice are largely determined by the understanding of the CKO of what would work, users' perceptions of what they need and the organizational work culture (Tiwana 2000:396).

According to Tiwana (2000:401), the CKO as a technologist is inter alia responsible for:

- the creation of enterprise-wide skills and knowledge directories;

- creation of channels for exchange of documents and other codified forms of explicit knowledge;

- development and implementation of the intranet;

- support of group and collaborative work through collaborative technology tools and policies;

- development and implementation of tools for collaborative problem solving;

- building of repositories to store, for example 'lessons learned'; and

- enabling tacit knowledge transfer by means of tools, for example video conferencing.

'Knowledge management is about creating an environment in which knowledge is created, shared and utilised for business purposes' (TFPL 2001). Therefore, the CKO also plays the role of an environmentalist, that is, being responsible for the creation of an environment that stimulates and facilitates either arranged or chance discussions and conversations, or the development of events and processes that encourage more deliberate knowledge creation and exchange. These may include:

- the designing of spaces, such as designing offices and relaxation areas, or acquiring or furnishing retreats and learning centres;

- bringing together communities with common interests but who rarely interact with each other;

- building relationships with related leaders in human resources, information technology, the library, research and development, etc.;

- developing incentives to encourage knowledge sharing; and

- recognizing and promoting knowledge contributors who share knowledge across the enterprise.

CKOs are designers of knowledge directories, knowledge-based systems, knowledgeintensive business and management processes, knowledge exchange events, knowledgesharing physical spaces and knowledge protection policies (Earl 1999:5). Linking with the role of environmentalist, the CKO has to radically redesign performance measurement and executive appraisal systems to break down incentives centred on the individual, and visibly encourage collective knowledge development and sharing (Earl 1999:7).

Their designs are mostly conceptual in the sense that they work on an idea with a champion, contribute design suggestions and inject ideas from emerging knowledge management practices, just as a consultant or system analyst would (Earl 1999:5).

Gamble and Blackwell (2001:77) see the CKO as the facilitator of the knowledge-based enterprise. Therefore, the primary role of the CKO is to enable, not control, knowledge 
management (Tiwana 2000:399). To achieve this, the CKO:

- works with and through people;

- enlists sponsors, champions and do-ers; and

- supports clients in inventing, crafting and implementing their own ideas.

As a facilitator, the CKO identifies knowledge requirements to know what is needed by the enterprise and the tools that can help him or her to do the job effectively. The CKO also facilitates the development of learning networks and the organization of regular programmes of learning and knowledge sharing (Snyman 2001:278).

Gamble and Blackwell (2001:97) see the appointment of the CKO as a step in the direction of formal cultural change. According to Earl and Scott (2000:202), the CKO is seen as the latest change agent, following those who led total quality management (TQM), business process reengineering (BPR) and other similar initiatives. The CKO reads the enterprise's appetite for change and appreciates how to connect to and work along with other change initiatives. According to Tiwana (2001:400-401), CKO activities as a change agent can include:

- changing the corporate culture from that of defensive knowledge hoarding to knowledge sharing;

- removing technical and socio-cultural barriers to knowledge sharing, transfer, use and distribution; and

- aligning local knowledge creation activities in individual departments and teams with the long-term strategic knowledge vision of the enterprise.

The main aim of the CKO as a change agent is to persuade individuals and groups to accept and internalize the view that knowledge management matters and can yield a significant improvement of organizational performance. It is the role of the change agent to stimulate the idea that his or her position of CKO will not be a permanent position with an established function. Participants in the research project of Earl and Scott (2000:202) stated that if the $\mathrm{CKO}$ is a change agent he or she will quite rightfully be 'here today and gone tomorrow'.

To fulfil these roles, the CKO must possess certain knowledge and skills.

\section{Knowledge and skills of the CKO}

During surveys conducted by Earl and Scott (1997; 2000) and TFPL (1999a and 1999b), it was found that people in CKO positions represent various educational backgrounds and a wide variety of experience both inside and outside the business environment. They also often have several years of experience working in the current enterprise and therefore know the culture of the enterprise, and the key actors also know them (Earl and Scott 2002:197). The findings with regard to the educational backgrounds and experiences correlate with the findings obtained during an analysis of job advertisements for knowledge managers, specifically CKOs, in South Africa (Snyman 2001). A variety of qualifications and experience were identified. The most common qualification and experience required at a strategic level were a tertiary qualification (from a wide variety of disciplines, for example social sciences, economics, business, finance, etc.) and three to five years' management experience at senior managerial level. However, no specific qualification in knowledge management was required.

To fulfil the role of strategist, entrepreneur, evangelist, technologist, environmentalist, 
designer and change agent, a variety of skills and personal attributes are required. Apart from the general management skills such as leadership skills, project management skills, welldeveloped negotiation skills, etc., additional and unique skills are also required.

The CKO is responsible for the intangible assets of the enterprise and the creation of an enabled environment that facilitates the sharing of knowledge. Therefore, it is very important that the CKO should possess certain personal attributes. During research conducted in 1997 and reported in 1998, Earl and Scott (in Duffy 1998) found that, although CKOs have varied backgrounds, they often display similar personality traits - and those traits differentiated them as a group from other executives. In a follow-up study, Earl and Scott (2000:200) described CKOs as 'fun people having a fun time'. They are typically bubbly and enthusiastic, yet reflective and balanced. They tend to be eclectic and pragmatic, backing any idea that makes 'knowledge sense', and are ready to connect to other initiatives.

Neilson (2002) identifies the most important personal attributes that a CKO must bring to the job. These are indicated in Figure 2.

\section{Figure 2 Personal attributes of CKO (Neilson 2002)}

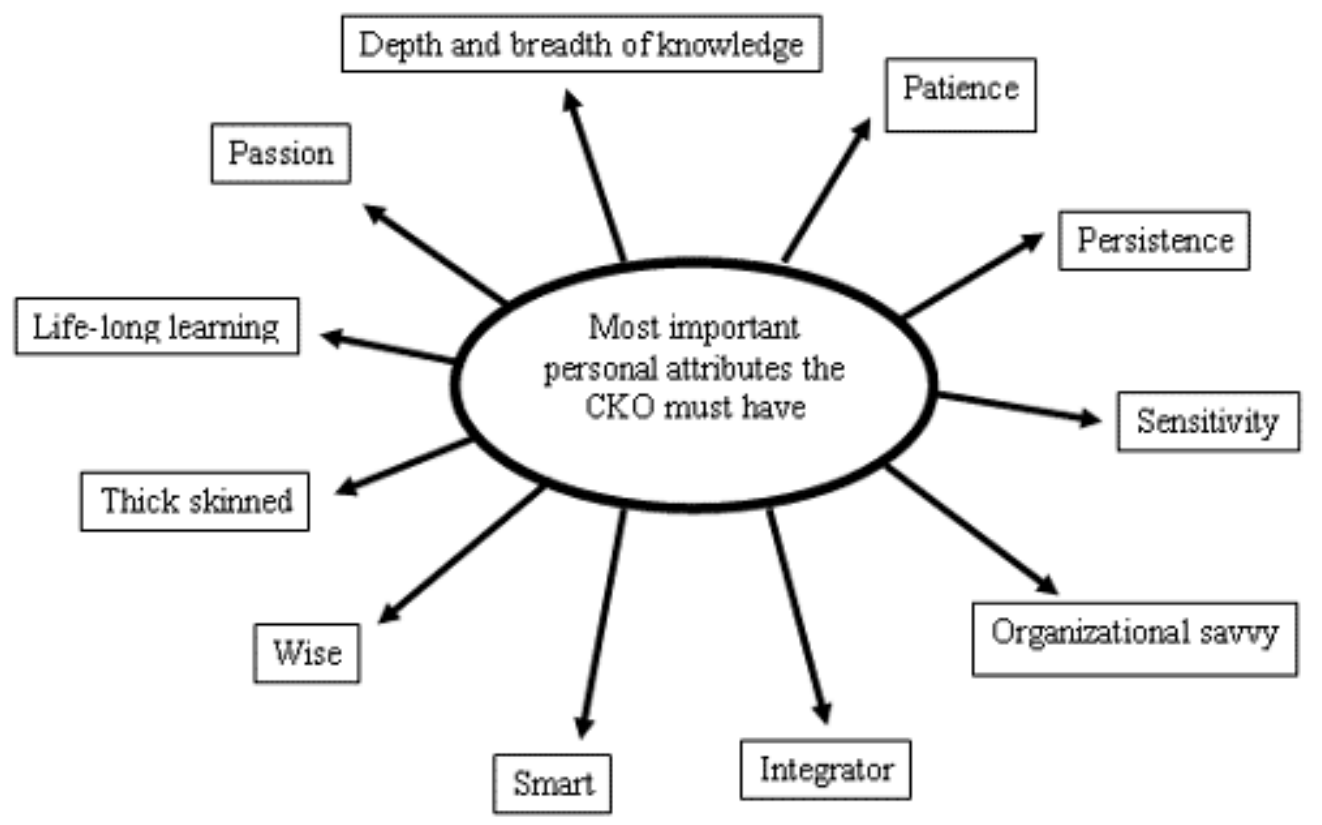

Although CKOs do not need any one personal trait in great abundance, all of them are necessary to a certain extent to build momentum behind the knowledge management initiatives.

Apart from having unique personality traits, CKOs also need unique competencies and skills to successfully fulfil the different roles. These are indicted in Figure 3.

Figure 3 Competencies and skills required (adapted from Neilson 2002) 


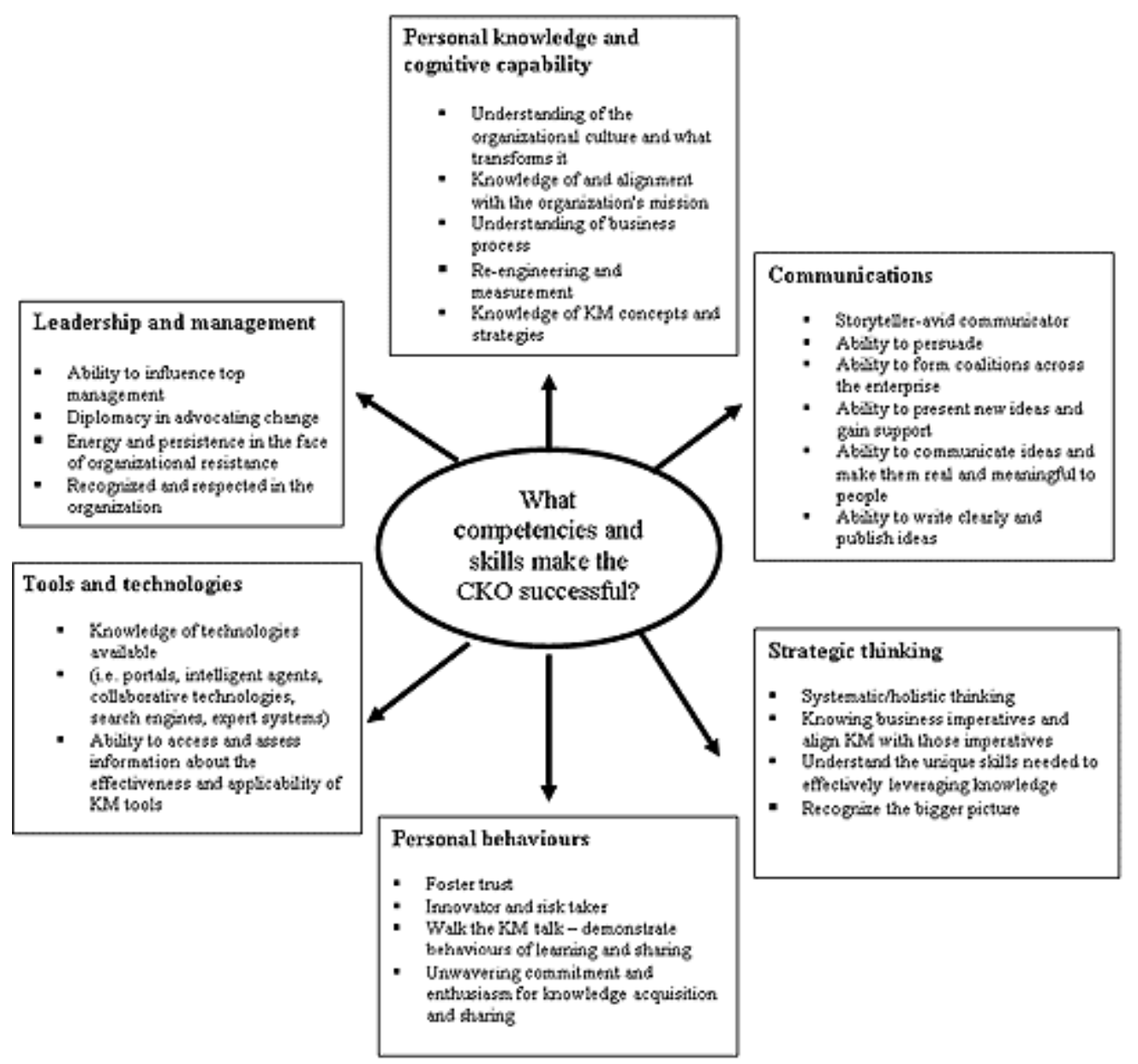

As a strategist, the CKO should be able to think strategically, systematically, conceptually and holistically. He or she should also understand the wider knowledge context and the organization's strategy (Skyrme 1997; TFPL 1999b). He or she must know the business imperatives and align knowledge management with these imperatives (Neilson 2002). The CKO must be able to grasp the implications of using knowledge management as a tool for corporate transformation. He or she must be able to see 'the bigger picture that the CEO has in mind, but also be able to translate it into action' (Earl and Scott 2000:197).

As an entrepreneur, the CKO should be able to present new ideas, focus on deliverable results and solicit support. To achieve this, the CKO must be a proactive self-starter, have the ability to work on own initiative and not be afraid of taking risks. He or she should maintain a balanced attitude toward achieving results, but should not get depressed by setbacks.

As an evangelist, the CKO should have an interest in knowledge management concepts and experience of knowledge management systems. He or she must articulate the knowledge management agenda and actively promote it.

As an environmentalist and designer, the CKO should be able to analyse situations, ask meaningful questions and propose solutions.

As a technologist, the CKO must understand which technologies can contribute to capturing, storing, exploring and, in particular, sharing knowledge. The CKO should be sufficiently informed about technology to:

- evaluate what works;

- judge when to adopt a technology; 
- appreciate the opportunities enabled; and

- assess any demanding implementation issues (Earl and Scott 2002).

As a facilitator, the CKO should be skilled at shaping the ideas of others and at knowing when to help. He or she should be sensitive to group dynamics and individual characters and should be able to tolerate small steps as much as big moves.

A combination of all the above-mentioned knowledge and skills equips the $\mathrm{CKO}$ as an excellent change agent. In addition, the CKO must be able to influence and diplomatically advocate change. It is important to be able to read the company's appetite for change and appreciate how to connect to and work with other change initiatives (Earl and Scott 2000:198).

\section{Findings of the interviews}

The three CKOs interviewed were all part of the top management team and reported directly to the CEO. One of the CKOs also had knowledge management responsibilities in the capability of executive director of people enablement on international level.

In two of the cases, persons involved in knowledge management initiatives in the organization filled the positions of CKO, while the third person was appointed from outside the organization. In all three cases, the reason for the position of CKO was to initiate, lead and enable the knowledge management strategy of the enterprise. These CKO positions were fairly new and had been established during the last two to six years.

The educational backgrounds and business experiences of the CKOs varied substantially. One had a BSc and MBA, worked in an IT environment for 10 years and lectured in Europe and South Africa. The second CKO had an undergraduate degree with mathematics and physics as major subjects as well as a BA Honours degree in communication. Before becoming $\mathrm{CKO}$, he was director of information product development and gained experience in managing research-based products. The third CKO had qualifications in library and information science and 24 years experience as librarian and information specialist. Two of the CKOs had additional qualifications in knowledge management or related areas such as content management and IT management. Each of the CKOs felt that their career experiences and attitude towards the job had groomed them for the position of CKO. However, all three confessed that every day was a learning experience. They also felt that they had gained their knowledge on the job and through experience.

In all three cases, the knowledge management position had no predecessors. In two of the cases, the CKOs had to develop their own job descriptions. This was achieved by taking risks, building relationships with stakeholders and colleagues, and by being alert to opportunities that may benefit the enterprise. These two CKOs were involved in various activities with regard to knowledge management. These activities included conducting research with regard to knowledge management, promoting knowledge management, determining the information and knowledge requirements of the enterprise and encouraging a culture of knowledge sharing by motivating staff to contribute to knowledge repositories. They were also responsible for working out strategies for harvesting, capturing and storing knowledge that were in line with the strategic objectives of the enterprise, as well as leveraging knowledge management for the benefit of the whole enterprise. Technical and organizational infrastructures were put into place so that knowledge could be transmitted, received and clearly understood. Their knowledge management approach was very balanced in the sense that their initiatives were focused on capturing the tacit knowledge via 
knowledge champions and making it explicitly available to others in the enterprise. Ongoing communication with information technologists and human resources executives existed to shape information sharing channels and capabilities.

During the interview, these two CKOs were very knowledgeable about the overall business objectives of their enterprises and the organizational culture. One can therefore conclude that they were contributing meaningfully to the company's strategy formulation.

The third CKO had a predefined job description. However, in contrast to the other two CKOs, it became evident during the interview that the third CKO's activities were limited to creating an awareness of knowledge management among stakeholders and colleagues, and that his primary role was that of an evangelist. This entailed the presentation of workshops and seminars on knowledge management, as well as the compilation of brochures and other documentation on knowledge management for distribution. No formal knowledge management system existed and no strategies were in place on how the enterprise should handle its intellectual capital.

As individuals, all three CKOs were open-minded and 'people persons'. They all fulfilled an important prerequisite for the successful management of knowledge, namely the ability to work with people. They were good communicators and possessed general managerial attributes such as leadership, interpersonal skills and project management skills. They had a high energy level that enabled them to manage the tasks associated with their jobs. They had a good understanding of how IT worked and how IT could be applied in facilitating networking and knowledge sharing.

Two of the CKOs indicated that the position of CKO was not a permanent position and might be absorbed in the organization under another title.

\section{Analysis of the findings}

In South Africa, as in other international countries, the CKO is a recent phenomenon. Only a few enterprises realize the value of appointing a senior executive responsible for managing one of their most important organizational assets, namely knowledge.

As found in the literature, the three CKOs also came from a wide range of professional backgrounds. Two of the CKOs knew the business and culture of the enterprises from personal experience and were established figures in their enterprises. The third CKO was appointed from outside the enterprise and still had to establish himself as an individual responsible for knowledge management in the enterprise.

Against the theoretical background and from the analysis of the findings, it is clear that two of the CKOs fulfilled almost of the roles identified in the literature study:

- Strategies were developed to dictate how the enterprise should handle its intellectual capital

- The knowledge management agenda was actively promoted

- Needs were determined and initiatives launched for the design and implementation of the knowledge architecture and infrastructure

- Knowledge-oriented connections, coordination and communication activities were facilitated

- The importance of IT as an enabler for information capturing, storage and dissemination was recognized. 
Although knowledge management in every organization is unique and the role and responsibilities of the CKO can be interpreted differently depending on the needs and environment of the enterprise, the third CKO still had a long way to go (taking the identified generic roles into consideration). His role was only that of an evangelist. All the other identified roles were not yet fulfilled. It can be attributed to various factors such as:

an environment in that did not yet realize the presence throughout the enterprise of information and knowledge that should be harvested and shared for the benefit of the enterprise;

- a corporate culture that did not support the sharing of knowledge;

- not being conversant with the business processes and internal politics - consequently he still had to build relationships with stakeholders and colleagues

- a job description that did not allow him the opportunity to apply initiatives of his own with regard to knowledge management; and

- a possible lack of support from management and employees for knowledge management initiatives.

If these constraints can be overcome, there should be no reason why this CKO cannot fulfil the roles as reflected in the literature.

\section{Conclusion}

The aim of this research was to investigate the perspective of the South African business world on the role and responsibilities of the CKO. Although the position of CKO is still unique and relatively new in South Africa, it compares very well with the findings in the literature. Although one cannot conclude that the findings of the three case studies are representative of the industry in general, it is clear that a strong awareness exists of the importance of the role that CKOs can play in South African enterprises. The findings further indicated that the three CKOs possessed the necessary competencies and skills to fulfil this important position.

\section{References}

Allee, V. 1997. The knowledge evolution: expanding organizational intelligences. Boston: Butterworth-Heinemann.

Bontis, N. 2002. The rising star of the chief knowledge officer. Ivey Business Journal (March/April):20-25.

Duffy, D. 1998. Knowledge champions: what does it take to be a successful CKO? CIO Enterprise Magazine (15 November). [Online]. Available WWW: http://www.cio.com/archive/enterprise/111598_ic_content.html (Accessed 16 April 2002).

Earl, M.J. 1999. What is a chief knowledge officer? Sloan management review via Gale Group. [Online]. Available WWW: http://lamb.cba.hawaii.edu:82/OldVersions/ What\%20is\%20a\%20Chief\%20Knowledge\%20Officer.htm (Accessed 24 June 2002). 
Earl, M.J and Scott, I.A. 2000. What do we know about CKOs? In Knowledge horizons: the present and the future of knowledge management. Boston: Butterworth-Heinemann: 195203.

Gamble, P.R. and Blackwell, J. 2001. Knowledge management: a state of the art guide. London: Kogan Page.

Herschel, R.T. and Nemati, H.R. 2000. Chief knowledge officer: critical success factors for knowledge management. [Online]. Available WWW: http://www.brint.com/members/online/20090319/CKO/

(Accessed 15 March 2002).

Morris, S., Meed, J. and Svensen, N. 1996. The intelligent manager: adding value in the information age. London: Prentice Hall.

Neilson, R.E. 2002. The competencies and skills of the CKO. [Online]. Available WWW: http://www.ndu.edu/irmc/km-cio_role/km-cio-role.htm

(Accessed 15 May 2002).

Nonaka, I. 1998. The knowledge-creating company. In: Harvard Business Review on knowledge management. Boston: Harvard Business School Publishing: 21-46.

Skyrme, D. 1997. Do you need a CKO? [Online]. Available WWW:

http//www.skyrme.com/insights/27cko.htm

(Accessed: 29 April 2002).

Snyman, R.M.M. 2001. Do employers really know what they want? Analysis of job advertisements for information and knowledge managers. Aslib Proceedings 53(7):273-281.

TFPL. 1999a. Skills for knowledge management: a briefing paper. London: TFPL.

TFPL. 1999b. Skills for knowledge management: building a knowledge economy. London: TFPL.

TFPL. 2001. Knowledge management state of the art workshop. Session 1: overview of KE and knowledge management. (Unpublished).

Tiwana, A. 2000. The knowledge management toolkit: practical techniques for building a knowledge management system. Upper Saddle River, NJ: Prentice Hall.

Wiig, K.M. 2000. Knowledge management: an emerging discipline rooted in a long history. In Knowledge horizons: the present and the future of knowledge management. Boston. Butterworth-Heinemann: 3-26.

\section{Disclaimer}

Articles published in SAJIM are the opinions of the authors and do not necessarily reflect the opinion of the Editor, Board, Publisher, Webmaster or the Rand Afrikaans University. The user hereby waives any claim he/she/they may have or acquire against the publisher, its suppliers, licensees and sub licensees and indemnifies all said persons from any claims, lawsuits, proceedings, costs, special, incidental, consequential or indirect damages, including damages for loss of profits, loss of business or downtime arising out of or relating to the user's use of the Website. 
ISSN 1560-683X

Published by InterWord Communications for the Centre for Research in Web-based Applications, Rand Afrikaans University 